\title{
EXACTLY SOLVABLE MODELS OF 2D DILATON GRAVITY
}

\author{
O. B. Zaslavskii \\ Astronomical Institute of Kharkiv V. N. Karazin National University, \\ 35 Sumska St., Kharkiv, 61022, Ukraine \\ e-mail: ozaslav@kharkov.ua \\ (Received October 13, 2006)
}

\begin{abstract}
We give the full list of types of static (homogeneous) solutions within a wide family of exactly solvable $2 \mathrm{D}$ dilaton gravities with the back-reaction of conformal fields. We discuss in detail the class of black hole solutions which includes the previously known ones as particular cases. For this class, the whole spacetime can be divided into different sheets with one horizon on each sheet between neighboring singularities with a finite value of dilaton field, the neighboring sheets being glued along the singularity. The position of singularities coincide with the values of dilaton in solutions with a constant dilaton field. Quantum corrections to the Hawking temperature vanish. We also consider a general approach to exactly solvable 2D dilaton cosmology. We find a rather rich class of everywhere regular solutions which exist practically in every type of the analyzed solutions. They exhibit different kinds of asymptotic behavior in the past and future, including inflation, superinflation, deflation, power expansion or contraction. In particular, for some models the dS spacetime with a time-dependent dilaton field is the exact solution of field equations. For some kinds of solutions the weak energy condition is violated independently of a specific model. We find also the solutions with a singularity which is situated in an infinite past (or future), so at any finite moment of a co-moving time the universe is singularity-free. It is pointed out that for some models the spacetime may be everywhere regular even in spite of infinitely large quantum back-reaction in an infinite past.
\end{abstract}

Key words: dilaton, two-dimensional gravity, quantum backreaction.

PACS number(s): 04.60.Kz, 98.80.Cq, 04.70.Dy

\section{INTRODUCTION}

Two-dimensional theories of gravity count on better understanding of the role of quantum effects in black hole physics in a more realistic four-dimensional case. In particular, a new insight was gained due to reducing the problem of black hole evaporation to the solution of differential equations of the semiclassical theory [1]. However, these equations remain too complicated in the sense that exact solutions cannot be found. This obstacle was overcome in the approach based on modifying the form of the semiclassical action in such a way that solvability is restored. The well-known example is the Russo-Susskind-Thorlacius (RST) model [2]. In particular, this enabled one to give exhaustire analysis of either geometry or thermodynamics of eternal black holes in the framework of RST dilaton gravity [3].

Examples of exactly solvable theories of dilaton gravity were discussed in $[4,5]$. As was shown by Kazama, Satoh and Tsuichiya (KST), these models as well as the RST one can be found as particular cases of the unified approach [6] based on the symmetries of the nonlinear sigma model. A number of other exactly solvable models were suggested later [7-10].

In the present paper we develop the general approach to exactly solvable 2D dilaton gravity theories with quantum back-reaction described by the Polyakov-Liouville action. The corresponding set of solutions includes all those known before. We dwell in detail on the solutions describing black holes and cosmological spacetimes. For black holes, we outline general features of their spacetime structure and thermodynamic properties. It turned out that, in spite of diversity of different forms of dilaton potentials, a wide class of exactly solvable models shares similar properties.

As far as the cosmological solutions in 2D dilaton gravity are concerned, a particular cosmological solution for such a system was found in [11] but it turned out that it suffers from the presence of unavoidable physical singularities. The interest in exact solutions in 2D semiclassical cosmology was further stimulated by the fact that the inclusion of back-reaction in some other models does cure the problem of cosmological singularities. This was observed in Ref. [12], where it was shown that this effect leads to a smooth transition between superinflation and FRW phases for some particular model and, thus, resolves the problem of the graceful exit (a brief review on this issue in the context of dilaton theory can be found in [13]). However, this result was achieved at the cost of putting the quantum coupling parameter $\kappa=\frac{\hbar(N-24)}{24}$ to negative values. Meanwhile, the quasiclassical approximation, within which the whole consideration was performed, implies $\hbar \rightarrow 0, N \rightarrow \infty$, that is $\kappa>0$. The improved model, free of unphysical restriction on $\kappa$, was analyzed in [14], [15] and was shown also to contain singularity-free solutions. These studies on 2D dilaton cosmology with everywhere regular solutions concerned only some special fixed models with the zero cosmological constant. In the present paper we relax the demand of having the zero cosmological constant (that, in the 
context of modern cosmology, looks more physical) and (i) give the full list of cosmological solutions within the exactly solvable models specified by the only relationship between the coefficient of the gravitation-dilaton action, (ii) find among them examples with everywhere bounded curvature.

\section{BASIC EQUATIONS}

Hereafter we restrict ourselves to semiclassical dilaton gravity with the back-reaction of conformal fields only. Consider the action

$$
I=I_{0}+I_{\mathrm{PL}}
$$

where

$$
I_{0}=\frac{1}{2 \pi} \int_{M} d^{2} x \sqrt{-g}\left[F(\phi) R+V(\phi)(\nabla \phi)^{2}+U(\phi)\right]
$$

and the Polyakov-Liouville action [18]

$$
I_{\mathrm{PL}}=-\frac{\kappa}{2 \pi} \int_{M} d^{2} x \sqrt{-g}\left[\frac{(\nabla \psi)^{2}}{2}+\psi R\right]
$$

responsible for the back-reaction of fields conformally coupled to gravity. Here the function $\psi$ obeys the equation

$$
\square \psi=R,
$$

where $\square=\nabla_{\mu} \nabla^{\mu}, \kappa=N / 24$ is the quantum coupling parameter, $N$ is the number of scalar massless fields, $R$ is a Riemann curvature. We omit the boundary terms in the action as we are interested only in field equations and their solutions.

From Eqs. (1)-(4) one can infer field equations (see below) which are valid for any gravitation-dilaton system of the kind under discussion. Meanwhile, our main goal is to analyze possible exactly solvable cases. The typical representative of the corresponding family reads

$$
\begin{aligned}
& F=\exp (-2 \phi)+2 \kappa(d-1) \phi \\
& V=4 \exp (-2 \phi)+2(1-2 d) \kappa+4 C\left(e^{-2 \phi}-\kappa d\right)^{2} \\
& U=4 \lambda^{2} \exp (-2 \phi)
\end{aligned}
$$

$\lambda$ and $d$ are constants. If $C=0$, this model turns to the one suggested in [10]. In turn, it includes different particular known models. For example, in the case $d=0$ one obtains the model suggested in [9], if $d=1 / 2$ it coincides with the RST model [2]. Meanwhile, the family of exactly solvable models under discussion in our paper is wider than (5), including it only as a particular class.

Let us return to the issue of field equations in the generic case. Varying the action with respect to a metric gives us $\left(T_{\mu \nu}=2 \frac{\delta I}{\delta g^{\mu \nu}}\right)$ :

$$
T_{\mu \nu} \equiv T_{\mu \nu}^{(0)}-T_{\mu \nu}^{(\mathrm{PL})}=0
$$

where

$$
\begin{gathered}
T_{\mu \nu}^{(0)}=\frac{1}{2 \pi}\left\{2\left(g_{\mu \nu} \square F-\nabla_{\mu} \nabla_{\nu} F\right)-U g_{\mu \nu}\right. \\
\left.+2 V \nabla_{\mu} \phi \nabla_{\nu} \phi-g_{\mu \nu} V(\nabla \phi)^{2}\right\} \\
T_{\mu \nu}^{(\mathrm{PL})}=\frac{\kappa}{2 \pi}\left\{\partial_{\mu} \psi \partial_{\nu} \psi-2 \nabla_{\mu} \nabla_{\nu} \psi+g_{\mu \nu}\left[2 R-\frac{1}{2}(\nabla \psi)^{2}\right]\right\}
\end{gathered}
$$

Variation of the action with respect to $\phi$ gives rise to the equation

$$
R \frac{d F}{d \phi}+\frac{d U}{d \phi}=2 V \square \phi+\frac{d V}{d \phi}(\nabla \phi)^{2} .
$$

In general, field equations cannot be solved exactly and function $\psi$, dilaton $\phi$ and metric depend on both time-like $(t)$ and space-like $(\sigma)$ coordinates: $\psi=\psi(t, \sigma)$, $\phi=\phi(t, \sigma)$. In what follows we restrict ourselves to such a kind of solutions that $\psi$ can be expressed in terms of $\phi$ only: $\psi=\psi(\phi)$. We will see that this leads to the existence of the Killing vector. On the other hand, as all static or homogeneous solutions depend on one variable, one may exclude it and express $\psi$ in terms of $\phi$. Thus, the assumption $\psi=\psi(\phi)$ turns out to be equivalent to the static or homogeneous character of solutions.

Let us take the trace of Eqs. (6)-(8) and Eq. (9). Denoting

$$
\tilde{F} \equiv F-\kappa \psi, U \equiv \Lambda e^{\int d \phi \omega}
$$

we get

$$
U=\square \tilde{F}
$$

$$
\begin{aligned}
& A_{1} \square \phi+A_{2}(\nabla \phi)^{2}=0, \\
& A_{1}=(u-\kappa \omega) \psi^{\prime}+\omega u-2 V, \\
& A_{2}=(u-\kappa \omega) \psi^{\prime \prime}+\omega u^{\prime}-V^{\prime},
\end{aligned}
$$

where $u \equiv F^{\prime}$ and prime throughout the paper denotes a differentiation with respect to $\phi$. For arbitrary coefficients $A_{1}(\phi), A_{2}(\phi)$ Eq. (12) cannot be solved exactly. This can be done, however, under some restrictions on the form of the coefficients $A_{1}, A_{2}$. Let us demand that

$$
A_{1}=(u-\kappa \omega) \chi^{\prime}, A_{2}=(u-\kappa \omega) \chi^{\prime \prime}
$$

where $\chi=\chi(\phi)$ and $\square \chi=0$. Then it follows that $\psi=\psi_{0}+\chi$, where

$$
\psi_{0}^{\prime}=\frac{2 V-\omega u}{u-\kappa \omega},
$$

which enables us to find at once $\psi_{0}$ in terms of the known functions $u, V, \omega$ by direct integration. Demanding that both equations in (13) be consistent with each other, we obtain the restriction on the action coefficients

$$
u^{\prime}(2 V-\omega u)+u\left(u \omega^{\prime}-V^{\prime}\right)+\kappa\left(\omega V^{\prime}-2 V \omega^{\prime}\right)=0
$$

This equation can be solved: 


$$
V=\omega\left(u-\frac{\kappa \omega}{2}\right)+C(u-\kappa \omega)^{2},
$$

where $C$ is a constant.

The fact that function $\psi$ is defined up to a function whose Laplacian vanishes is explained by Eq. (4) which is, in fact, the definition of $\psi$. The presence of $\chi$ reveals itself in the nature of quantum state (see below). Eq. (16) is just the condition obtained in [16], so account for $\chi$ does not generate new types of exactly solvable models but extends the set of solutions within these models.

With Eq. (11) taken into account, the field equations (6)-(8) can be rewritten in the form

$$
\left[\xi_{1} \square \phi+\xi_{2}(\nabla \phi)^{2}\right] g_{\mu \nu}=2\left(\xi_{1} \nabla_{\mu} \nabla_{\nu} \phi+\xi_{2} \nabla_{\mu} \phi \nabla_{\nu} \phi\right)
$$

where $\xi_{1}=\frac{d \tilde{F}}{d \phi}, \xi_{2}=\frac{d^{2} \tilde{F}}{d \phi^{2}}-\tilde{V}, \tilde{V}=V-\frac{\kappa}{2}\left(\frac{d \psi}{d \phi}\right)^{2}$. Let us multiply this equation by the factor $\zeta$ chosen in such a way that $\xi_{2} \zeta=\frac{d\left(\xi_{1} \zeta\right)}{d \phi}$. Then Eq. (17) turns into

$$
g_{\mu \nu} \square \mu=2 \nabla_{\mu} \nabla_{\nu} \mu,
$$

where by definition $\mu^{\prime}=\xi_{1} \zeta$. This equation takes the same form as eq. (2.24) from [3] and entails the same general conclusion about the existence of the Killing vector $l_{\alpha}=\varepsilon_{\alpha}^{\beta} \mu_{, \beta}$. In the present section we consider the case when the Killing vector is time-like everywhere that gives rise to static solutions and mainly concentrates on black hole ones.

It is convenient to work in the conformal gauge

$$
d s^{2}=g\left(-d t^{2}+d \sigma^{2}\right)
$$

where, in accordance with the choice of the Killing vector, $g=g(\sigma)$ and does not depend on a time-like coordinate $\sigma$. In gauge (19) the curvature

$$
R=-g^{-1} \frac{\partial^{2} \ln g}{\partial \sigma^{2}} .
$$

Eq. (11) takes the form

$$
\Lambda e^{\eta}=\frac{\partial^{2} \tilde{F}}{\partial \sigma^{2}} g^{-1}, \eta=\int d \phi \omega .
$$

Now for any function $f(\sigma)$ we have $\square f=g^{-1} \frac{\partial^{2} f}{\partial^{2} \sigma}$ whence it is clear that $\chi=\gamma \sigma$, where $\gamma$ is a constant. Thus, we have

$$
\psi=\psi_{0}+\gamma \sigma
$$

where $\psi_{0}$ is defined according to (14). It follows from (4) that

$$
g=e^{-\psi-a \sigma}=e^{-\psi_{0}-\delta \sigma},
$$

where $a$ is a constant, $\delta=\gamma+a$. After a simple rearrangement the (00) and (11) field equations (6), (17) with the metric in the conformal gauge (19) are reduced to one equation

$$
\xi_{1} \frac{d^{2} \phi}{d \sigma^{2}}+\xi_{2}\left(\frac{d \phi}{d \sigma}\right)^{2}-\xi_{1} g^{-1} \frac{d g}{d \sigma} \frac{d \phi}{d \sigma}=0 .
$$

It is convenient to split the coefficients in Eq. (24) into two parts singling out the term which is built up with the help of $\psi_{0}: \xi_{1}=\xi_{1}^{(0)}-\kappa \gamma \frac{d \sigma}{d \phi}, \xi_{2}=\xi_{2}^{(0)}-\kappa \gamma \frac{d^{2} \sigma}{d \phi^{2}}+$ $\kappa\left[\frac{d \eta}{d \phi} \gamma \frac{d \sigma}{d \phi}+\frac{1}{2}\left(\gamma \frac{d \sigma}{d \phi}\right)^{2}\right]$,

$$
\begin{aligned}
& \xi_{1}^{(0)}=\frac{d \tilde{F}^{(0)}}{d \phi}, \quad \xi_{2}^{(0)}=\frac{d^{2} \tilde{F}^{(0)}}{d \phi^{2}}-\tilde{V}^{(0)}, \\
& \tilde{F}^{(0)}=F-\kappa \psi_{0}, \quad \tilde{V}^{(0)}=V-\frac{\kappa}{2}\left(\frac{d \psi_{0}}{d \phi}\right)^{2} .
\end{aligned}
$$

Then Eq. (24) takes the form

$$
\begin{aligned}
\xi_{1}^{(0)} \frac{d^{2} \phi}{d \sigma^{2}} & +\xi_{2}^{(0)}\left(\frac{d \phi}{d \sigma}\right)^{2}+\xi_{1}^{(0)} \frac{d \phi}{d \sigma}\left(\frac{d \psi_{0}}{d \sigma}+\delta\right) \\
& =\kappa \gamma\left(\delta-\frac{\gamma}{2}\right) .
\end{aligned}
$$

Let us multiply this equation by the factor $s$ so that $\xi_{2}^{(0)} s=\frac{d\left(\xi_{1}^{(0)} s\right)}{d \phi}, s=\exp \left[\frac{\left(\xi_{2}^{(0)}-\xi_{1}^{(0)^{\prime}}\right)}{\xi_{1}^{(0)}}\right]=$ $\exp \left[-\tilde{V}^{(0)} / \xi_{1}^{(0)}\right]$.

Then eq. (26) can be cast into the form

$$
\frac{d z}{d \sigma}+z\left(\frac{d \psi_{0}}{d \sigma}+\delta\right)=\kappa \gamma\left(\delta-\frac{\gamma}{2}\right) s,
$$

where $z=s \xi_{1}^{(0)} \frac{d \phi}{d \sigma}=s \frac{d \tilde{F}^{(0)}}{d \sigma}$. It follows from (14) and (16) that

$$
\begin{gathered}
\psi_{0}=\eta+2 C H, \\
g=e^{-\eta-2 C H-\delta \sigma}
\end{gathered}
$$

and

$$
\tilde{F}^{(0)}=H(1-2 \kappa C),
$$

where $H=F-\kappa \eta$. If $\Lambda \neq 0$, the metric function (up to the constant factor) is equal to

$$
\begin{gathered}
g=\frac{e^{-\delta \sigma-2 C H}}{U}, \\
H=F-\kappa \ln U+\text { const. }
\end{gathered}
$$

We obtain from $(16),(25),(28),(30)$

$$
\begin{aligned}
\tilde{V}^{(0)} & =(1-2 \kappa C) H^{\prime}\left(\omega+C H^{\prime}\right), \\
\xi_{1}^{(0)} & =(1-2 \kappa C) H^{\prime},
\end{aligned}
$$

whence

$$
s=e^{-\eta-C H} .
$$

Then after a simple rearrangement Eq. (27) gives rise to

$$
\frac{d^{2} H}{d \sigma^{2}}+C\left(\frac{d H}{d \sigma}\right)^{2}+\delta \frac{d H}{d \sigma}=\alpha,
$$


where

$$
\alpha=\kappa \gamma\left(\delta-\frac{\gamma}{2}\right) /(1-2 \kappa C) .
$$

It is convenient to introduce a new variable $\rho$, where $|\rho|=e^{C H}$. Then we have the linear equation

$$
\frac{d^{2} \rho}{d \sigma^{2}}+\delta \frac{d \rho}{d \sigma}=\alpha C \rho
$$

One can seek a solution in the form $\rho \sim e^{\beta \sigma}$, whence we obtain

$$
\beta^{2}+\delta \beta-\alpha C=0
$$

This equation is quadratic and has two roots $\beta_{1}, \beta_{2}$. Depending on their properties, one can classify all possible types of solutions and describe their properties. In a natural way, the solutions fall into three different classes: I (both $\beta_{1}, \beta_{2}$ are real, $\left.\beta_{1} \neq \beta_{2}\right)$; II $\left(\beta_{1}, \beta_{2}\right.$ are real, $\beta_{1}=\beta_{2}$ ), III (roots are complex, $\beta_{1}=\beta_{2}^{*}$ ). We describe the results below.

\section{A GENERAL CASE. TYPES OF SOLUTIONS}

It is convenient to cast the solutions of Eq. (35) into uniform formulas:

$$
C H=C H_{0}-\frac{\delta \sigma}{2}+\ln |f|,
$$

where the function obeys the equation

$$
\frac{d^{2} f}{d \sigma^{2}}=f \varepsilon^{2}, \varepsilon^{2}=\frac{\delta^{2}}{4}+\alpha C .
$$

We get the following different cases.

$$
\mathrm{I}_{a} \cdot \varepsilon^{2}>0, f=\frac{\operatorname{sh} \varepsilon \sigma}{\varepsilon} ; \mathrm{I}_{b}: f=\frac{\operatorname{ch} \varepsilon \sigma}{\varepsilon} ;
$$

$\mathrm{II}_{a}: \varepsilon=0, f=\sigma ; \mathrm{II}_{b}: f=1$;

III: $\varepsilon^{2} \equiv-\varkappa^{2}<0, f=\frac{\sin \varkappa \sigma}{\varkappa}$.

It follows from (21) that

$$
\frac{\Lambda C}{1-2 \kappa C}=e^{2 C H_{0}} z,
$$

where $z=1$ for the $\mathrm{I}_{b}$ case, $z=0=\Lambda$ for $\mathrm{II}_{b}$ and $z=-1$ in cases $\mathrm{I}_{a}, \mathrm{II}_{a}$, III.

The Riemann curvature reads the following.

$\mathrm{I}_{a}, \mathrm{II}_{a}, \mathrm{III}$ :

$$
R=\frac{U C}{1-2 \kappa C}\left[2+\frac{\omega}{C H^{\prime}}-\frac{1}{C^{2} H^{\prime}}\left(\frac{\omega}{H^{\prime}}\right)^{\prime} q^{2}\right] .
$$

$\mathrm{I}_{b}:$

$$
R=\frac{U C}{1-2 \kappa C}\left[2+\frac{\omega}{C H^{\prime}}+\frac{1}{C^{2} H^{\prime}}\left(\frac{\omega}{H^{\prime}}\right)^{\prime} q^{2}\right]
$$

$\mathrm{II}_{b}$ :

$$
R=\frac{e^{\eta+2 C H_{0}}}{1-2 \kappa C} \frac{1}{C^{2} H^{\prime}}\left(\frac{\omega}{H^{\prime}}\right)^{\prime} \frac{\delta^{2}}{4} .
$$

Here $q=\left(\frac{d f}{d \sigma}-\frac{\delta}{2} f\right)$.
In a similar way, we get the general structure of the expression for quantum stresses. Two nonzero components of quantum stresses are connected for conformal fields by the well known relationship $T_{0}^{0(\mathrm{PL})}+T_{1}^{1(\mathrm{PL})}=\frac{\kappa R}{\pi}$ (see eq. (8)). Here we list the component $T_{1}^{1(\mathrm{PL})}$ only. One obtains from (8), (28), (29), (36):

$$
\begin{aligned}
& T_{1}^{1}=-\frac{1}{4 \pi g}\left[\kappa\left(\frac{\partial \psi_{0}}{\partial \sigma}+2 \delta\right) \frac{\partial \psi_{0}}{\partial \sigma}+2 \alpha(1-2 \kappa C)\right], \\
& \frac{\partial \psi_{0}}{\partial \sigma}=\left(\frac{\omega}{C H_{\phi}^{\prime}}+2\right) \frac{q}{f}
\end{aligned}
$$

whence

$$
\begin{gathered}
T_{1}^{1(\mathrm{PL})}=-\frac{\kappa}{4 \pi} \frac{|U C|}{1-2 \kappa C} Z, \\
Z=\left(\frac{q \omega}{C H^{\prime}}+2 f^{\prime}\right)^{2}-(\delta-\gamma)^{2} f^{2},
\end{gathered}
$$

except the case $\mathrm{II}_{b}$, when

$$
T_{1}^{1(\mathrm{PL})}=-\frac{\kappa}{4 \pi} e^{2 C H_{0}+\eta} Z,
$$

$Z=\frac{\delta^{2}}{4}\left(\frac{\omega}{C H^{\prime}}\right)^{2}-(\delta-\gamma)^{2}$.

\section{BLACK HOLES}

It is clear from the formulas listed above that there exists a significant variety of different types of solutions. We restrict ourselves by the simplest but physically relevant cases and dwell mainly on black hole solutions. (The complete classification can be obtained as direct counterpart of that for the homogeneous time-dependent solution which is considered below in the section "Cosmological solutions". Therefore, to avoid duplication, in the present section we omit it.) Apart from this, we consider the case when the quantum fields are in the HartleHawking state, so that we have the state of thermal equillibrium between a black hole and quantum fields, the stress-energy tensor of these fields being finite on the horizon as usual. The reservations made in the previous sentence are meaningful since in $2 \mathrm{D}$ theories there are exact self-consistent solutions for which the state interpolates between the Hartle-Hawking and Boulware state, the geometry being regular notwithstanding the fact that the stresses diverge [21].

Let $C=0=\alpha=\gamma, \omega=$ const. For convenience, we choose $\omega=-2$ to facilitate comparison with standard models. Then it follows from (35) that

$$
H=H_{0}+D \exp (-\delta \sigma) .
$$

If, instead of the conformal coordinate $\sigma$ one uses the Shwarzschild one, the metric can be cast into the from (17)-(35) that

$$
d s^{2}=-g d t^{2}+g^{-1} d x^{2}
$$


where

$$
\begin{gathered}
g=A \int_{\phi_{h}}^{\phi} d \tilde{\phi} \frac{\partial \mu}{\partial \tilde{\phi}} e^{\psi(\tilde{\phi})-\psi(\phi)} \\
x=B^{-1} \mu, \mu=\int^{\phi} d \tilde{\phi} \frac{\partial H}{\partial \tilde{\phi}} e^{\int^{\tilde{\phi}} d \phi^{\prime} p\left(\phi^{\prime}\right)}, \\
p=-\frac{\tilde{V}}{H^{\prime \prime}}
\end{gathered}
$$

Now we can make general conclusions about the structure of spacetime. Curvature (19) can be rewritten in the Schwarzschild coordinates as $R=-\frac{d^{2} g}{d x^{2}}$. Then, we obtain

$$
R=\frac{4 \lambda^{2}}{H^{\prime}}\left[\frac{\omega\left(H-H_{h)}\right.}{H^{\prime}}\right]^{\prime} \exp \left(\int^{\phi} d \tilde{\phi} \omega\right)
$$

Let, by definition, $H^{\prime}\left(\phi_{c}\right)=0, x_{c}=x\left(\phi_{c}\right)$. Near $\phi_{c}$ the function $H^{\prime} \propto \phi-\phi_{c}, x-x_{c} \propto\left(\phi-\phi_{c}\right)^{2}$ and, in general, $R \propto\left(\phi-\phi_{c}\right)^{-3} \propto\left(x-x_{c}\right)^{-3 / 2}$. The exceptional case arises when $\phi_{c}=\phi_{h}$. Then the above expression in square brackets is finite and $R \propto\left(\phi-\phi_{c}\right)^{-1} \propto\left(x-x_{c}\right)^{-1 / 2}$. Thus, singularity becomes weaker but does not disappear. Such a behavior, found earlier for the RST model [3], is inherent to any model under consideration.

Thus, the metric possesses singularities in the points $\phi=\phi_{c}$ where $H^{\prime}=0$. The spacetime splits into intervals between zeros of $H^{\prime}$ which can be viewed as different sheets that generalizes the corresponding feature of the RST model [3]. Within each of them function $H^{\prime}$ does not alter its sign, so function $H(\phi)$ is monotonic and the equation $H=H_{h}$ has only one root. Then, according to (19), there is only one horizon at $\phi=\phi_{h}$ on every sheet between any two singularities with a finite value of $\phi$. In principle, it may happen that on a sheet between infinity and a singularity nearest to it there exists an additional horizon due to the factor $e^{-\psi}$ in which case the coordinate $x$ calculated according to (19) takes, generally speaking, a finite value in this limit. To obtain the maximally extended analytical continuation of spacetime, one is led to accept the possibility of complex dilaton field values [19]. We will not, however, discuss such possibilities further. For the RST model there exist only two sheets but, depending on the properties of function $H(\phi)$, the number of sheets in a general case can be made arbitrary. Any two neighboring sheets are separated by a singularity located at $\phi=\phi_{c}$.

From eqs. (50), (52) we obtain the formula for the Hawking temperature $T_{H}=\left(\frac{d g}{d x}\right)_{x=x_{h}}$ which turns out surprisingly simple:

$$
T_{H}=(2 \pi)^{-1} \lambda
$$

and does not acquire quantum corrections. Moreover, as this temperature is a constant, it turns out that all horizons present in the solution have the same temperature. Both properties generalize a similar feature of the RST model [3]. In the latter case $H \propto e^{\omega \phi}$.
Apart from the solutions discussed above, there is one more class of them. It is seen from eq. (12) that this equation turns into identity when $\phi=$ const $\equiv \phi_{0}$. For such solutions field Eq. (11) gives us $U=-\kappa R$. Substituting it into eq. (9) we have $R \tilde{F}^{\prime}=0$ where we have taken into account that $H^{\prime}=u-\kappa \omega$. This means that nontrivial solutions $(R \neq 0)$ exist only for the values of the dilaton field $\phi_{0}=\phi_{c}$. Let me recall that this is just the point where the curvature for solutions described by eq.(19) diverges. This gives a nontrivial interplay between two branches of solutions, also found for the particular case of the RST model [3]: the values of the dilaton field for constant dilaton solutions coincide with the singularity of non-constant ones. In particular, it follows from the content of the present paragraph that the class of models under discussion does not contain constant dilaton solutions with two horizons found in [20]. It is not surprising since the latter solutions exist only under the presence of an electromagnetic field which is now absent.

\section{COSMOLOGICAL SOLUTIONS}

In the section "Basic equations" we pointed out that the theories of gravity under discussion admit the Killing vector. Up till now, we discussed the case when this vector is time-like, the corresponding solutions being static. In the present section we consider the case when the Killing vector associated with the spacetimes under discussion has the space-like character, so we will be dealing with homogeneous cosmological solutions. In contrast to the black hole case, here we consider in general variety non-zero parameters introduced above. They turn out to be essential for physically relevant solutions. Homogeneous counterparts of static solutions have a similar structure considered above but they have quite different meaning.

\section{A. Particular cases and limiting transitions}

The solutions obtained depend on several parameters. In what follows it is assumed that the dilaton is not identically constant. Quantities $\Lambda$ and $C$ enter the definition of the action coefficients: $\Lambda$ is the "amplitude" of the potential $U$ of a generic model according to eq. (10), while parameter $C$ defines coefficient $V$ of an exactly solvable one (16). Meanwhile, quantities $\delta$ and $\alpha$ are the parameters of the solutions of field equations, they do not enter the action but characterize the different solutions for the same model. Let us denote symbolically $[C, \Lambda](\delta, \alpha)$ solutions with the given parameters for a given action, where it is supposed that the values of parameters differ from zero, unless otherwise stated explicitly. Consider first the case

$$
\text { B. } C=0
$$

Now 


$$
\psi_{0}=\eta, g=e^{-\eta-\delta t}
$$

In the cases $[0,0](0, \alpha)$ and $[0,0](\delta, 0)$ it turns out that field equations are mutually inconsistent, so these cases cannot be realized.

$$
\begin{gathered}
\text { 1. Type }[0,0](0,0) \\
H=A t, g=e^{-\eta}, R=-\frac{A^{2}}{H^{\prime}}\left(\frac{\omega}{H^{\prime}}\right)^{\prime} e^{\eta}, \\
T_{0}^{0(\mathrm{PL})}=\frac{\kappa}{4 \pi} A^{2} \frac{\omega^{2} e^{\eta}}{H^{\prime 2}}>0 .
\end{gathered}
$$

Here $A$ is an arbitrary constant.

Below we will mainly concentrate on the potentials of the form

$$
H=e^{-2 \phi}
$$

and

$$
H=e^{-2 \phi}-\kappa d \phi, d>0 .
$$

The condition $d>0$ ensures that $H^{\prime}(\phi)<0$ and does not change the sign.

Example.

Let, for definiteness, $A<0$,

$$
\omega=-2 n, 0<n<2 .
$$

Then for model (57) we have a superinflation with the exact solution

$$
\begin{aligned}
& a \backsim(-\tau)^{p}, \quad p=-\frac{n}{2-n}, \quad R \backsim \frac{1}{\tau^{2}}, \\
& \phi=\phi_{0}+\frac{1}{n-2} \ln |\tau|,
\end{aligned}
$$

( $\phi_{0}$ is a constant). Hereafter we use for shortness dimensionless time $\tau$. The case $n=2$ corresponds to de Sitter spacetime with $a \backsim \exp (\tau)$.

A More interesting situation arises for case (58). Then for $\tau \rightarrow-\infty$ eqs. (60) hold asymptotically $(\phi \rightarrow-\infty)$, whereas for $\tau \rightarrow \infty(\phi \rightarrow \infty)$ we have

$$
a \backsim \tau, \quad R \backsim \tau^{-s}, \quad s=\frac{2 n+2}{n} .
$$

Thus, we have a graceful exit from superinflation to the FRW (Friedmann-Robertson-Walker) phase. This solution generalizes the previous result of [15] (which holds for $n=1$ ) to the case of an arbitrary $0<n<2$.

Let now $n=2$. Then for the model of the type (58) $a \backsim \exp (\tau), \phi \backsim \frac{\tau}{2} \rightarrow-\infty$ at $\tau \rightarrow-\infty$ and $a \backsim \tau$ and $R \backsim \tau^{-3}$ at $\tau \rightarrow \infty$. Thus, we obtain the graceful exit from inflation to FRW (Friedmann-Robertson-Walker) phase.

For an arbitrary $n$ the curvature

$$
R \backsim \frac{\exp [-(2 n+2) \phi]}{[2 \exp (-2 \phi)+\kappa d]^{3}}
$$

remains finite everywhere for any $1 \leq n \leq 2$.
It is instructive to compare this with previous papers on semiclassical exactly solvable cosmological models. Actually, the solution considered in [22] corresponds just to the case $[0,0](0,0)$ and a particular model (58) for $n<0$. In [22] there was obtained the power asymptotic behavior at the beginning of expansion near $\tau=0$, where $a \backsim \tau$ that it is rather difficult to call "inflation" (as it was called in [22]). Moreover, as the spacetime is geodesically incomplete in the case considered there (parameter $\tau$ having the meaning of co-moving time is finite), it seems that this is not a cosmological solution at all but, rather, it describes a transition from the homogeneous to the static, when an observer crosses an event horizon of a white hole where $a=g=0$. Therefore, the finiteness of the curvature on the horizon tells nothing about the possible existence of a singularity which may lie behind the horizon.

Compare now our results with those in [15]. Using $a=\exp (\phi)$ from (56), we obtain (taking for simplicity in (56) $A=-1$ ) for model (58)

$$
\tau=\kappa d e^{\phi}-2 e^{-\phi}=\kappa d a-2 a^{-1} .
$$

Solving eq. (63) with respect to $e^{\phi}$, we obtain

$$
a=\frac{1}{\kappa d}\left(\sqrt{\tau^{2}+2 \kappa d}+\tau\right) \text {. }
$$

This corresponds to eq. (17) of [15] (a reader should bear in mind that in [15] by definition $\kappa=\frac{N \hbar}{12}$, while we use $\left.\kappa=\frac{N \hbar}{24}\right)$. Choosing the values of constants in [15], $\alpha=1$, $\beta=0$ (this can be always done without loss of generality by a proper shift and recalling variable $\tau$ ) and $d=1$ in our paper, we achieve full coincidence. The curvature is everywhere bounded. For $\tau \rightarrow \infty R \backsim \tau^{-4}$ that agrees with (61) in the case $n=1$.

It is also worth commenting on some general properties of the geometry and quantum stresses for the kind of solution under discussion, generalizing the previous observations made for particular representatives of the models studied in $[15,17]$. If $\omega=$ const (that is indeed the case for the most popular dilaton potentials and was used in the above consideration), $R=\frac{A^{2} \omega H^{\prime \prime} e^{\eta}}{H^{\prime 3}}$. For the nonsingular models, which represent the most interest, $H^{\prime}$ does not vanish. If, for definiteness, $H^{\prime}<0$ and $\omega<0$, we obtain that $R>0$. Taking into account (20), we obtain that in any kind of such a model $\frac{d^{2} a}{d \tau^{2}}>0$ : the universe is ever accelerating.

The WEC (weak energy condition) is violated $\left(T_{0}^{0(\mathrm{PL})}>0\right)$ for any kind of the solution $[0,0](0,0)$. On the other hand, it turns out that for any solution of the type $[0,0](0,0)$ the classical part of the energy density vanishes. Indeed, let us write down field equations in the form

$$
T_{c l . \mu}^{\nu}=T_{\mu}^{\nu(\mathrm{PL})}+T_{q \mu}^{\nu(\phi)} \equiv \theta_{\mu}^{\nu},
$$

where the term $T_{q \mu}^{\nu(\phi)}$ is obtained by varying the part of the term in the gravitation-dilaton part that depends on $\kappa$ explicitly. By definition, the term $T_{c l . \mu}^{\nu}$, when expressed in terms of the metric and dilaton according to (6), does 
not contain $\kappa$. Then, calculating (6) with the condition of exact solvability (16) taken into account, we obtain that $\theta_{0}^{0}=0$.

\section{Type $[0,0](\delta, \alpha)$}

$$
\begin{aligned}
& H=\frac{\alpha}{\delta} t, a=\exp \left(-\frac{\eta}{2}-\frac{\delta^{2}}{2 \alpha} H\right), \\
& R=-\frac{\alpha^{2}}{\delta^{2}} \frac{1}{H^{\prime}}\left(\frac{\omega}{H^{\prime}}\right)^{\prime} \exp \left(\eta+\frac{\delta^{2}}{\alpha} H\right), \\
& T_{0}^{0(\mathrm{PL})}=\frac{\exp \left(\eta+\frac{\delta^{2} H}{\alpha}\right)}{4 \pi} \alpha\left[\kappa\left(\frac{\alpha \omega}{\delta H^{\prime}}+2 \delta\right) \frac{\omega}{\delta H^{\prime}}+2\right] .
\end{aligned}
$$

If $\alpha=A \delta$ and $\delta \rightarrow 0$, while $A$ is kept fixed, (66) turns into (56). If $H^{\prime}<0 H^{\prime \prime}>0$ and $\omega<0$, curvature $R>0$, so similarly to the $[0,0](0,0)$ case we obtain accelerating spacetimes only.

If $\delta>0$ and $\alpha>0$ (this is just the case discussed in [15]) the curvature diverges at $t \rightarrow \infty$. Let now $\delta>0$, $\alpha \equiv-\kappa \mu<0$. Then for model (58) with $\omega=-2 n$ curvature $R$ is bounded everywhere if $q \equiv 2 n+2-2 p \geq 0$, where $p \equiv \frac{d \delta^{2}}{2 \mu}$, otherwise it diverges at $\phi \rightarrow \infty$.

Example.

For model (58) we obtain at $t \rightarrow-\infty: a \backsim(-\tau) \rightarrow \infty$, $\exp (-2 \phi) \backsim(-t) \rightarrow \infty, R \backsim|\tau|^{-2}(\ln |\tau|)^{-2}$. Thus, $R$ always remains bounded at $\tau \rightarrow-\infty$ independent of the value of $q$.

At $t \rightarrow \infty \phi \backsim \frac{\mu}{d \delta} t, a \backsim \exp \left[t \frac{\mu}{\delta d}(1-p)\right]$. If $p \leq 1$, the spacetime is geodesically complete since $\tau \rightarrow \infty$. Consider first the case $p<1$. Then $a \backsim \tau, \phi \backsim \ln \tau$, $R \backsim \exp (-q \phi) \backsim \exp \left(-\frac{\mu}{\delta d} q t\right) \backsim \tau^{-s}, s=q(1-p)^{-1}>0$. Thus, we have a FRW-like expansion. Let now $p=1$. Then $a \rightarrow 1$ and the spacetime becomes asymptotically flat.

The above solutions $[0,0](0,0)$ and $[0,0](\delta, \alpha)$ represent what was called, correspondingly, "first and second branches" in [15].

For model (58) with $\omega=-2$ it is convenient to rewrite the scale factor in (66) in the form

$$
a=\exp \left(\phi-\frac{\delta t}{2}\right)=\exp \left[\phi\left(1+\frac{\delta^{2} d \kappa}{2 \alpha}\right)-\frac{\delta^{2}}{2 \alpha} e^{-2 \phi}\right]
$$

Introducing notation $\xi=e^{-2 \phi}$ and choosing the values of constants $\delta^{2}=2 \mu>0, \alpha=\kappa \mu, d=1$ we have

$$
a=\xi^{-1} \exp \left(-\frac{\xi}{\kappa}\right)
$$

that coincides in this particular case with Eq. (24) of [15].

It is worth stressing that the choice of the sign of $\alpha$ (while keeping $\delta$ positive) has a crucial effect on the asymptotic behavior of the geometry. For $\alpha>0$ the solution $[0,0](\delta, \alpha)$ contains a singularity in agreement with [15] whereas for $\alpha<0$, as we saw above, the geometry is everywhere regular.

$$
\text { 3. Type }[0, \Lambda](\delta, \alpha)
$$

$$
\begin{gathered}
H=h(t)=H_{0}+\frac{\alpha}{\delta} t+D e^{-\delta t} \\
R=\frac{e^{\eta}}{H^{\prime}}\left[\omega \Lambda-\left(\frac{\omega}{H_{\phi}^{\prime}}\right)_{\phi}^{\prime}\left(\frac{\alpha^{2}}{\delta^{2}} e^{\delta t}-2 \alpha D+D^{2} \delta^{2} e^{-\delta t}\right)\right] \\
T_{0}^{0(\mathrm{PL})}=\frac{1}{4 \pi} e^{\eta+\delta t}\left\{2 \alpha+\kappa\left(\frac{\alpha}{\delta}-D \delta e^{-\delta t}\right)\right. \\
\left.\times \omega H^{\prime}-1\left[2 \delta+\left(\frac{\alpha}{\delta}-D \delta e^{-\delta t}\right) \omega H^{\prime}-1\right]\right\} \\
D \delta^{2}=-\Lambda .
\end{gathered}
$$

Let $\Lambda<0, D>0, \delta<0, \alpha>0$. By a proper shift in $t$, one can always achieve $D=1$. Then there exists some point $t_{0}$ at which $h^{\prime}\left(t_{0}\right)=0$. Let $H=e^{-2 \phi}$, $\omega=-2$. Then at $t \rightarrow \infty, \phi \rightarrow-\infty$ we have a flat spacetime, $\phi=-\frac{|\delta| t}{2}, g \rightarrow 1, R \sim \kappa \phi e^{2 \phi} \rightarrow 0$. Consider $t \rightarrow t_{0}$, where $h^{\prime}\left(t_{0}\right)=0$ and choose $H_{0}=\frac{\alpha}{\delta^{2}}\left(\ln \frac{\alpha}{\delta^{2}}-1\right)$, so that $h\left(t_{0}\right)=0, h=\alpha \frac{\left(t-t_{0}\right)^{2}}{2}+\ldots$ near $t_{0}$. Then $\phi \rightarrow \infty, g \rightarrow \infty$, the proper time $\tau \sim \ln \left(t-t_{0}\right) \rightarrow-\infty$, $a \backsim \exp (-\tau), \phi \backsim-\tau$. We obtain de Sitter space undergoing deflation.

There also exists the second branch of solutions the: $-\infty<t<t_{0}$. For this branch we have inflation in future: $a \backsim \exp (\tau) \rightarrow \infty$. In the infinite past $\exp (-2 \phi) \backsim \frac{\alpha}{|\delta|}|t|$, $a \backsim-\tau, R \backsim \tau^{-2} \ln ^{-2}|\tau|$. The point $\tau=0$ is singular.

If $\alpha>0, \delta>0$, the universe in the second branch starts from the flat state and ends up with eternal inflation.

$$
\begin{gathered}
\text { 4. Type }[0, \Lambda](\delta, 0) \\
H=H_{0}+D e^{-\delta t}, \quad g=e^{-\eta-\delta t}, \\
R=\frac{U}{H^{\prime}}\left[\omega+\left(H-H_{0}\right)\left(\frac{\omega}{H^{\prime}}\right)^{\prime}\right], \\
T_{0}^{0(\mathrm{PL})}=\frac{\kappa \omega U}{4 \pi H^{\prime}}\left[2-\frac{\omega\left(H-H_{0}\right)}{H^{\prime}}\right] .
\end{gathered}
$$

Example.

$$
H=A e^{-\phi}+e^{-2 \phi}, A>0 .
$$

Let $\delta=-|\delta|<0, H_{0} \equiv-e^{|\delta| t_{0}}<0, D>0, \Lambda<0$, $\omega=-2$. We may achieve $D=1$. Then $t_{0}<t<\infty$. When $t \rightarrow t_{0} \phi \rightarrow \infty, a \backsim\left(t-t_{0}\right)^{-1} \backsim \exp (-\tau), \tau \rightarrow-\infty$. At future $\tau \rightarrow \infty, \phi=-\frac{1}{2}|\delta| t \rightarrow \infty, a \rightarrow 1$. Thus, we have the graceful exit from deflation to the flat spacetime.

In the particular case $A=2$ it is possible to obtain an explicit solution for the whole region. Solving (71) with respect to $e^{-\phi}$, we find

$$
\begin{gathered}
e^{-\phi}=\sqrt{1+H_{0}+D e^{-\delta t}}-1, \\
a=\frac{\exp \left(-\frac{\delta t}{2}\right)}{\sqrt{1+H_{0}+D e^{-\delta t}}-1} .
\end{gathered}
$$

The solution $[0, \Lambda](0,0)$ proves to be inconsistent with field equations. 


\section{Type $[0, \Lambda](0, \alpha)$}

$$
\begin{aligned}
& H=\frac{\alpha t^{2}}{2}+H_{0}, g=e^{-\eta}, \Lambda=-\alpha=\frac{\kappa \gamma^{2}}{2}>0 \\
& R=\frac{U}{H^{\prime}}\left[\omega+2\left(\frac{\omega}{H^{\prime}}\right)^{\prime}\left(H-H_{0}\right)\right], \\
& T_{0}^{0(\mathrm{PL})}=-\frac{U}{2 \pi}\left[1+\left(\frac{\omega}{H^{\prime}}\right)^{2} \kappa\left(H-H_{0}\right)\right] .
\end{aligned}
$$

This kind of solutions exists due to quantum effects $(\kappa \neq 0)$ only.

\section{Examples.}

$H=e^{2 \phi}-A e^{-2 \phi}, \omega=-2, A>0$. Then we have the solution which behaves asymptotically as $a \backsim \exp (\tau)$ at $\tau \rightarrow-\infty$ and $a \backsim \exp (-\tau)$ at $\tau \rightarrow \infty$. Thus, we have the everywhere regular solution which starts at the inflation phase, passes through the maximum value of $a$ and ends up with deflation.

$H=e^{\phi}-c e^{-3 \phi}, \omega=-2, c>0$. Then at $\tau \rightarrow-\infty$ $a \backsim(-\tau)^{-2}$ and at $\tau \rightarrow \infty a \backsim \tau^{-2}$. Thus, superinflation is changed to superdeflation with everywhere bounded curvature.

\section{C. $C \neq 0$}

\section{Type $[C, \Lambda](0,0)$.}

Then

$$
\begin{aligned}
& H=H_{0}+C^{-1} \ln \left|\frac{t}{t_{0}}\right|, \quad g=e^{-\eta}\left(\frac{t_{0}}{t}\right)^{2}=\frac{\text { const }}{|U| t^{2}} \\
& a=\frac{a_{0}}{|t| \sqrt{|U|}}, \\
& R=\frac{U}{1-2 \kappa C}\left[\frac{\omega}{H^{\prime}}+2 C-\left(\frac{\omega}{H^{\prime}}\right)^{\prime} \frac{1}{H^{\prime} C}\right] \\
& \Lambda C=(1-2 \kappa C) t_{0}^{-2}>0 \\
& T_{0}^{0(\mathrm{PL})}=\frac{\kappa U C}{4 \pi(1-2 \kappa C)}\left(2+\frac{\omega}{C H^{\prime}}\right)^{2}>0
\end{aligned}
$$

where $a_{0}, t_{0}$ are constants. Thus, the weak energy condition (WEC) is always violated for this type of solutions.
It is worth noting that the dependence of the metric on the dilaton $g(\phi)$ for this kind of solutions coincides with that for $[0,0](\delta, \alpha)$, provided $C=\delta^{2} / 2 \alpha$.

On the other hand, the dependence of the dilaton on the proper time actually coincides with that for the $[0,0](0,0)$ since in both cases

$$
\tau(\phi)=\text { const } \int d \phi H^{\prime}(\phi) \exp (-\eta / 2) .
$$

Consider model (57) with $H_{0}=0, U=\Lambda e^{-4 \phi}, \Lambda$, $C>0,-\infty<t<t_{0}$. Then

$$
\begin{gathered}
\tau=-\tau_{0} \ln \left(\ln \left|\frac{t}{t_{0}}\right|\right) \\
a=a_{1} \exp \left[-\exp \left(-\frac{\tau}{\tau_{0}}\right)+\frac{\tau}{\tau_{0}}\right] \\
a_{1}=\frac{a_{0} C}{\left|t_{0}\right| \sqrt{\Lambda}}, \quad \tau_{0}=a_{1}\left|t_{0}\right| \\
e^{-2 \phi}=C^{-1} \exp \left(-\frac{\tau}{\tau_{0}}\right)
\end{gathered}
$$

The exact expression for the curvature is

$$
R=\frac{2}{\tau_{0}^{2}}\left[1+\exp \left(-\frac{\tau}{\tau_{0}}\right)+\exp \left(-\frac{2 \tau}{\tau_{0}}\right)\right] .
$$

Thus, we have a remote singularity in the infinite past and the inflationary stage in an infinite far future.

The next example is model (58) with $C>0, H_{0}=0$, $U=\Lambda e^{-2 \phi}, 0<t<\infty$. Then it follows from (77) that at $t \rightarrow 0, \tau \rightarrow-\infty$,

$$
a \sim(-\tau)^{s}, s=1+\kappa C d, R \backsim \tau^{-2}, \phi \backsim \ln |\tau|,
$$

at $t \rightarrow \infty, \tau \rightarrow \infty$

$$
a \sim \tau^{-1} \exp \left(-\tau^{2}\right) \rightarrow 0, R \sim \tau^{2}, \phi \backsim-\ln \tau .
$$

Thus, the universe starts from the flat spacetime and infinite scale factor and exhibits power contraction, at the far future we have a remote singularity.

$$
\begin{aligned}
& \text { 2. Type }[C, \Lambda](\delta, 0): \\
& e^{C H}=e^{C H_{0}}\left|1+D C e^{-\delta t}\right|, \quad g=e^{-\eta} \frac{\left[e^{C\left(H-H_{0}\right)} \nu-1\right]}{D C} e^{-2 C H}, \\
& R=\frac{U}{(1-2 \kappa C)}\left\{2 C+\frac{\omega}{H^{\prime}}+\frac{1}{C H^{\prime}}\left(\frac{\omega}{H^{\prime}}\right)^{\prime}\left[\nu e^{C\left(H-H_{0}\right)}-1\right]\right\}, \quad \frac{\Lambda}{(1-2 \kappa C)}=-e^{2 C H_{0}} D \delta^{2}, \\
& T_{0}^{0(\mathrm{PL})}=\frac{U C}{4 \pi} \frac{\kappa}{1-2 \kappa C}\left(\frac{\omega}{C H^{\prime}}+2\right)\left\{2+\frac{\omega}{C H^{\prime}}\left[1-\nu e^{C\left(H-H_{0}\right)}\right]\right\}, \\
& \nu=\operatorname{sign}\left(1+D C e^{-\delta t}\right) .
\end{aligned}
$$


Example

Let us take the same model as in (73) and choose $D=1, C>0, \delta<0, \frac{\exp \left(-C H_{0}\right)-1}{C} \equiv \frac{\exp \left(C e^{|\delta| t_{0}}-1\right)}{C} \equiv$ $\exp \left(|\delta| t_{1}\right)$. Then $t_{1}<t<\infty$. At $t \rightarrow t_{1}$ the solution has the same asymptotic behavior as for $C=0$ that is deflation $a \backsim \exp (-\tau)$. However, the behavior at $t \rightarrow \infty$ changes drastically as compared to $[0, \Lambda](\delta, \alpha)$. Indeed, now we have $H \backsim C^{-1}|\delta| t$ instead of $H \backsim e^{|\delta| t}$. We have, instead of the flat spacetime (inherent to the $C=0$ case) the singularity at the finite value of $\tau=\tau_{0}$, where

$$
\begin{aligned}
& g \backsim t^{-1} \exp (-|\delta| t), \quad a \backsim \tau_{0}-\tau \rightarrow 0, \\
& R \backsim t^{-1} \exp (|\delta| t) \backsim\left(\tau_{0}-\tau\right)^{-2} \ln ^{-2}\left(\tau_{0}-\tau\right) .
\end{aligned}
$$

One more example.

$\delta<0, D=1, C=-|C|, H_{0}=0, H$ is the same as in (73). Consider the cosmological solution

$$
\begin{gathered}
\exp (-|C| H)=\exp (|\delta| t)-1, \\
g=\frac{\exp (|\delta| t)}{U[\exp (|\delta| t)-1]^{2}},
\end{gathered}
$$

defined in the region $0<t<t_{0}$, where $\exp \left(|\delta| t_{0}\right)=2$. Then at $t \rightarrow t_{0} e^{-\phi} \backsim t_{0}-t, a \backsim\left(t_{0}-t\right)^{-1} \backsim \exp (\tau) \rightarrow$ $\infty$, so we have inflation. At $t \rightarrow 0 e^{-2 \phi} \backsim-\ln t$,

$$
\begin{aligned}
& a \backsim \frac{1}{t \sqrt{(-\ln t)}} \backsim(-\tau)^{-1} \exp \left(\tau^{2}\right), \\
& \tau \rightarrow-\infty, \quad R \backsim \tau^{2} .
\end{aligned}
$$

Thus, we have the remote singularity in an infinite past.

The type of solutions under discussion possesses one more interesting pecularity. Usually, in the standard inflationary cosmology, the scalar field is assumed to be approaching the constant, the corresponding effective potential playing the role of a cosmological constant. Now we will see that the family of solutions described above contains a qualitatively different possibility: $(\nabla \phi)^{2} \neq 0$, but de Sitter space-time $(\mathrm{dS})$ is an exact solution of field equations. If the dilaton depends only on time, so does the metric and we get an exponentially growing scale factor.

Indeed, let $\omega=\eta=0, U=\Lambda=$ const. For example, according to (16) with $F=e^{\phi}$, we have $V=C e^{2 \phi}$. Then it follows from $(87)$ that for the solution $[C, \Lambda](\delta, 0)$ the Riemann curvature

$$
R=\frac{2 \Lambda C}{1-2 \kappa C}=-2 e^{C H_{0}} D C \delta^{2}
$$

is a constant. If $\Lambda C>0$, we obtain $R>0$, so we have the $2 \mathrm{D}$ dS metric. Let, for definiteness, $\delta=-|\delta|<0$. Then in the remote past $[1+D C \exp (-\delta t)]>0$. Making a proper shift in time and choosing $H_{0}=0$, we may achieve $D C=-1, R=2 \delta^{2}$. Then for $t<0$ we have from (87)

$$
e^{C H}=\left(1-e^{|\delta| t}\right), \quad g=\frac{e^{|\delta| t}}{\left(1-e^{|\delta| t}\right)^{2}},
$$

Integrating the expression for $\tau=\int d t a$, we see that $t=0$ corresponds to $\tau \rightarrow \infty$. We obtain the exact expressions in dimensionless variables

$$
\begin{aligned}
& |\delta| \tau=\ln \left[\frac{1+\exp \frac{|\delta| t}{2}}{1-\exp \left(\frac{|\delta| t}{2}\right)}\right], \\
& a=\sinh \hat{\tau}, \quad \hat{\tau}=|\delta| \tau, \quad 0<\tau<\infty
\end{aligned}
$$

the behavior of the dilaton is governed by the equation

$$
\exp (C H)=\cosh ^{-2}\left(\frac{\hat{\tau}}{2}\right) .
$$

The $2 \mathrm{D}$ dS metric can be viewed as the metric on the hyperboloid

$$
u^{2}-v^{2}+w^{2}=1
$$

embedded in the three-dimensional space

$$
d s^{2}=d u^{2}-d v^{2}+d w^{2} .
$$

There are three typical possibilities:

$$
\begin{gathered}
u=\sinh x \sinh \tau, v=\cosh x \sinh \tau, w=\cosh \tau, \\
d s^{2}=-d \tau^{2}+\sinh ^{2} \tau d x^{2}, \\
u=\sin z \cosh t, v=\sin z \sinh t, w=\cos z, \\
d s^{2}=-d t^{2} \sin ^{2} z+d z^{2}, \\
u=\frac{\rho^{2}-t_{1}^{2}-1}{2 t_{1}}, \quad v=\frac{\rho^{2}-t_{1}^{2}+1}{2 t_{1}}, \\
w=\frac{\rho}{t_{1}}, \quad t_{1}= \pm e^{-\tau_{1}}, \\
d s^{2}=-d \tau_{1}^{2}+e^{2 \tau_{1}} d \rho^{2} .
\end{gathered}
$$

Usually, it is metric (103) which is considered in the theory of inflation. In the region $|w|<1,|u|>|v|$ metric (103) can be reduced to the static form (101). This is impossible for metric (99), for which $|u|<|v|,|w|>1$. The geometry, described by (94) is geodesically incomplete since at $\tau \rightarrow 0$ we have a horizon separating a black hole and cosmological (non-static) regions. Once the point $\tau=0$ is achieved from positive values, the system enters the static region.

Eqs. (94), (95) describe the inflationary regime at $\tau \rightarrow \infty$ provided $C H(\phi) \rightarrow-\infty$. If $\omega \neq 0$, but $\omega \rightarrow 0$ asymptotically, the inflationary regime can be considered as an approximation.

$$
\text { 3. Type }[C, \Lambda](0, \alpha)
$$

It can be obtained directly from types I or III by putting $\delta=0$. 


\section{Type $[C, 0](\delta, \alpha)$ :}

$C H=\beta_{ \pm} t, \quad g=\exp \left(-\eta \mp 2 \varepsilon C H \beta_{ \pm}^{-1}\right)$,

$R=-\frac{\beta_{ \pm}^{2}}{C^{2} H^{\prime}}\left(\frac{\omega}{H^{\prime}}\right)^{\prime} e^{\eta \pm 2 \varepsilon C H \beta_{ \pm}^{-1}}$,

$T_{0}^{0(\mathrm{PL})}=\frac{1}{4 \pi} \exp \left(\eta \pm 2 \varepsilon \frac{C H}{\beta_{ \pm}}\right)$

$\times\left\{\kappa \beta_{ \pm}\left(2+\frac{\omega}{C H^{\prime}}\right)\left[2\left(\beta_{ \pm}+\delta\right)+\frac{\beta_{ \pm} \omega}{C H^{\prime}}\right]+2 \alpha(1-2 \kappa C)\right\}$,

$\beta_{ \pm}=-\frac{\delta}{2} \pm \varepsilon$. The solution $[C, 0](0, \alpha)$ does not bring any qualitative new features and can be obtained directly from (104) by putting $\delta=0$.

The equivalence between $[0,0]\left(\delta_{1}, \alpha_{1}\right)$ and $[C, 0](\delta, \alpha)$, $\delta_{1}=2 \varepsilon, C= \pm \frac{\alpha_{1} \beta_{ \pm}}{\delta_{1}}$.

The dependence $g(\phi)$ coincides with that for $[0,0](0,0)$ provided $\delta^{2} / \alpha=2 \varepsilon C / \beta$. The dependence $\tau(\phi)$ coincides with that for $[0,0](\delta, \alpha)$, provided $\delta^{2} / \alpha=2 \varepsilon C / \beta$.

5. Type $[C, 0]\left(\delta_{0}, \alpha\right)$, where $\delta_{0}^{2} \equiv-4 \alpha C$ :

$$
\begin{gathered}
H=H_{0}-\frac{\delta_{0} t}{2 C}, g=e^{-\eta}, \\
R=\frac{\alpha}{C H^{\prime}}\left(\frac{\omega}{H^{\prime}}\right)^{\prime} e^{\eta}, \\
T_{0}^{0(\mathrm{PL})}=\frac{\alpha}{4 \pi}\left(2-\frac{\kappa \omega^{2}}{C H^{\prime 2}}\right) e^{\eta} .
\end{gathered}
$$

This dependence of the metric and dilaton on time coincides with $[0,0](0,0)$, provided $A=-\delta_{0} / 2 C$.

The solution $[C, 0](\delta, 0)$ can be obtained from (104) directly by putting $\alpha=0$.

\section{REGULAR SOLUTIONS WITH INFINITELY STRONG BACK-REACTION}

It is common belief that quantum effects can destroy classical singularity, thus leading to everywhere regular spacetime. This was confirmed explicitly for the models considered in $[12,15]$, where it was shown that it is the finite quantum parameter $\kappa$ that removes the singularity, inherent to the classical limit $\kappa=0$. In the present paper, where also examples with everywhere bounded curvature were found, it is essential that (at least in some cases) this behavior is due to the nonzero coefficient $d$ or $\kappa$ (they appear as a product $d \kappa$ in model (58)). Thus, quantum back-reaction can give rise to a regularity of the metric.

Meanwhile, in 2D dilaton gravity there exist cases when this back-reaction becomes so strong that the contribution from $T_{\mu}^{\nu(\mathrm{PL})}$ diverges by itself. One could expect these divergencies to destroy the regularity of the spacetime: in this sense not only the absence of backreaction but also too strong a back-reaction would seem incompatible with the regularity of the geometry. Nevertheless, sometimes the geometry can remain finite even in spite of divergencies in $T_{\mu}^{\nu(\mathrm{PL})}$. This was shown for black hole solutions [21]. For cosmological ones these results could not be applied directly since the cosmological counterpart of black holes considered there would have a finite $\tau$ (an analogue of the proper length) and, thus, would be geodesically incomplete. However, now we will see, using the materials of the previous sections, that a combination of divergent quantum stresses with regular geometry is possible for cosmological spacetimes as well.

Let us reconsider the solution $[0,0](\delta, \alpha)$ for the model discussed after Eq. (66), with $p<1$. For $t \rightarrow \infty, \phi \rightarrow \infty$, $\tau \rightarrow \infty, H^{\prime} \rightarrow-\kappa d=$ const, $R \backsim \exp (-q \phi), T_{0}^{0(\mathrm{PL})} \backsim$ $\exp [(2-q) \phi]$. If $q=2 n+2-2 p \geq 0$ but $q<2$, the curvature remains bounded but the energy density of quantum fields diverges. This is indeed possible and is compatible with the condition of the geodesic completeness, provided $n<p<1<n+1$.

Let us now turn to the type $[0, \Lambda](0, \alpha)$. It follows from eq. (76) and the following formulas that in the limits $\tau \rightarrow \pm \infty$, when $\phi \rightarrow-\infty, T_{0}^{0(\mathrm{PL})} \backsim U \backsim \exp (-2 \phi)$ becomes infinite. Meanwhile, the metric approaches de Sitter one with the constant curvature.

The fact that in an infinite past (or infinite future) quantum effects could be enormously large, is not in disagreement in intuitive expectations about possible contribution from quantum effects near classical singularity. We see that these effects cannot (at least, for some kinds of models) destroy the regular character of the spacetime.

\section{SUMMARY}

Thus, we considered a rather wide family of exactly solvable models of $2 \mathrm{D}$ dilaton gravity with back-reaction of conformal fields, which includes previously known particular models of this kind, and enumerated all possible types of static or homogeneous solutions that appear in this family.

For black hole solutions, we singled out that in a wide class of them (which, in particular, includes the RST model) in which different solutions share common properties. Namely, the set of possible solutions is split to different branches so that all spacetimes for $\phi \neq$ const can be divided to separate sheets with one and only one horizon on every sheet between two neighboring singularities with finite $\phi_{c}$ (plus, perhaps, additional horizons due to $\phi=\infty$ or $\phi=-\infty)$, different sheets are glued in singular points; all horizons on different sheets share the same temperature.

We also analyzed string-inspired models of dilaton cosmology, in which all coefficients are simple combinations of exponential and linear functions of dilaton $\phi$. We showed that practically any subset of solutions may describe a singularity-free universe, which starts in an infinite past and expands or contracts forever. We found also that in some cases these kinds of solution can occupy the intermediate place between completely regular and singular spacetimes, representing remote singularities. This means that singularity exists but it lies in an 
infinite past (or infinite far future) with respect to any event, so no observer hits it.

Quantum effects are crucial for the most part of the considered cosmological solutions since they affect qualitatively the behavior of the system at $\phi \rightarrow \infty$ or $\phi \rightarrow-\infty$ in the initial or final state. In this sense, quantum back-reaction turns out to be a powerful tool of removing singularities inherent to classical solutions. Moreover, in some cases even divergencies of quantum stresses in the initial or final state $(\tau \rightarrow-\infty$ or $\tau \rightarrow \infty)$ do not spoil the regularity of the geometry. For some particular types, such as $[0, \Lambda](0, \alpha)$, the solution does not exist at all without account for quantum terms.

It is worth paying attention to the non-standard version of the inflation scenario which is contained in the type $[C, \Lambda](0,0)$. Being geodesically incomplete, it represents the part of the de-Sitter world that expands exponentially fast asymptotically, but in doing so the dilaton field also depends on time, while the effective potential $U(\phi)$ (which usually plays the role of a cosmological term with $\phi=$ const) is absent.

We restricted ourselves to the simplest models. However, the approach, once the condition of exact solvability is respected, applies to any kind of models within this class, so the results admit further extension.

\section{ACKNOWLEDGMENTS}

To write the paper for the issue of JPS in honour of Prof. I. O. Vakarchuk, I decided to try to follow the pattern which Ivan Oleksandrovych shows to us so successfully: the application of quantum theory to relativistic systems with special emphasis on exactly solvable models.
[1] C. G. Callan, S. Giddings, J. A. Harvey, A. Strominger, Phys. Rev. D 45, R1005 (1992); T. Banks, A. Dabholkar, M. R. Douglas, M. O'Loughlin, Phys. Rev. D 45, 3607 (1992).

[2] J. G. Russo, L. Susskind, L. Thorlacius, Phys. Rev. D 46, 3444 (1992); Phys. Rev. D 47, 533 (1992).

[3] S. N. Solodukhin, Phys. Rev. D 53, 824 (1996).

[4] A. Bilal, C. G. Callan, Nucl. Phys. B 394, 73 (1993).

[5] S. P. de Alwis, Phys. Rev. D 46, 5429 (1992).

[6] Y. Kazama, Y. Satoh, A. Tsuichiya, Phys. Rev. D 51, 4265 (1995).

[7] G. Michaud, R. C. Myers, preprint, gr-qc/9508063.

[8] A. Fabbri, J. G. Russo, Phys. Rev. D 53, 6995 (1995).

[9] S. Bose, L. Parker, Y. Peleg, Phys. Rev. D 52, 3512 (1995).
[10] J. Cruz, J. Navarro-Salas, Phys. Lett. B 375, 47 (1996).

[11] F. D. Mazzitelli, J. G. Russo, Phys. Rev. D 47, 4490 (1993).

[12] S.-J. Rey, Phys. Rev. Lett. 77, 1929 (1996).

[13] S.-J. Rey, preprint, hep-th/9609115.

[14] S. Bose, preprint, hep-th/9704175.

[15] S. Bose, S. Kar, Phys. Rev. D 56, 4444 (1997).

[16] O. B. Zaslavskii, Phys. Rev. D 59, 084013 (1999).

[17] W. T. Kim, M. S. Yoon, Phys. Lett. B 423, 231 (1998).

[18] A. M. Polyakov, Phys. Lett. B 103, 207 (1981).

[19] R. Balbinot, A. Fabbri, Class. Quant. Grav. 13, 2457 (1996).

[20] O. B. Zaslavskii, Phys. Lett. B 424, 271 (1998).

[21] O. B. Zaslavskii, Phys. Rev. D 61, 64002 (2000).

[22] W. T. Kim, V. S. Yoon, Phys. Rev. D 58, 08014 (1998).

\title{
ТОЧНО РОЗВ'ЯЗУВАНІ МОДЕЛІ ДВОВИМІРНОЇ ДИЛАТОННОЇ ІРАВІТАЦІЇ
}

\author{
О. Б. Заславський \\ Інститут астрономї̈ Харківсъкого \\ національного університету імені В. Н. Каразіна, \\ вул. Сумсъка, 35, Харків, 61022, Україна
}

\begin{abstract}
Ми подаємо повний перелік типів статичних (однорідних) розв'язків у широкому сімействі точно розв'язуваних моделей двовимірної дилатонної гравітації з урахуванням зворотної реакції конформних полів. Докладно обговорюємо клас чорнодірних розв'язків, що містить раніше знайдені. Для цього класу повний простір-час розпадається на різні листи з горизонтом на кожному з них між найближчими сингулярностями, так що сусідні листи склеюються вздовж сингулярностей. Положення сингулярностей відповідає величині дилатона для розв'язків зі сталим дилатонним полем. Квантові поправки до температури Гокінга при цьому зникають. Ми також розглядаємо загальний підхід до точно розв'язуваних моделей у двовимірній дилатонній космології. Знайдено доволі загальний клас регулярних розв'язків, які існують практично в кожному типі аналізованих розв'язків. Вони демонструють різноманітні типи асимптотичної поведінки в минулому й майбутньому, включаючи інфляцію, суперінфляцію, дефляцію, статичне розширення або стиск. Зокрема для деяких моделей простір-час де Сіттера з дилатонним полем, що залежить від часу, є точним розв'язком рівнянь поля. Для деяких типів розв'язків слабка енергетична умова порушується незалежно від конкретної моделі. Ми також знаходимо розв'язок із сингулярністю, розташованою в нескінченному минулому (майбутньому), так що для будь-якого кінцевого моменту супутнього часу сингулярність буде відсутньою. Для деяких моделей простір-час може бути всюди регулярним, навіть за наявности нескінченно великої зворотної реакції квантових полів.
\end{abstract}

